\title{
SPHERICAL HARMONIC EXPANSION OF THE POISSON-SZEGÖ KERNEL FOR THE BALL
}

\author{
G. B. FOLLAND
}

ABSTRACT. By applying the theory of unitary spherical harmonics, we obtain an expansion of the Poisson-Szegö kernel for the unit ball in complex $n$-space in terms of Jacobi polynomials and hypergeometric functions.

1. Dictionary. We list here the various constants and special functions we will be using. The reader should proceed to $\$ 2$ and refer back here as necessary. For the relevant properties of these special functions we refer to the Bateman Manuscript Project [1].

(A) For $n=1,2,3, \cdots, \omega_{n}$ is the area of the unit sphere in $\mathbf{R}^{n}$ :

$$
\omega_{n}=2 \pi^{n / 2}\{\Gamma(n / 2)\}^{-1} \text {. }
$$

(B) For $k=0,1,2, \cdots$ and $n=2,3,4, \cdots, d(k ; n)$ is the dimension of the space of homogeneous harmonic polynomials of degree $k$ on $\mathbf{R}^{n}$ :

$$
d(k ; n)=(2 k+n-2)(k+n-3) !\{(n-2) ! k !\}^{-1} .
$$

(C) For $p, q=0,1,2, \cdots$ and $n=2,3,4, \cdots$, we set

$$
D(p, q ; n)=\frac{(p+q+n-1)(p+n-2) !(q+n-2) !}{p ! q !(n-1) !(n-2) !} .
$$

(D) For $\alpha, \beta>-1$ and $m=0,1,2, \cdots, P_{m}^{(\alpha, 3)}$ is the Jacobi polynomial of degree $m$ associated to $(\alpha, \beta)$ :

$$
P_{m}^{(\alpha, \beta)}(t)=\frac{(1-t)^{\alpha}(1+t)^{\beta}}{m ! 2^{m}} \frac{d^{m}}{d t^{\bar{m}}}\left[(1-t)^{x+m}(1+t)^{\beta+m}\right] .
$$

We have

Received by the editors December 19, 1973.

AMS (MOS) subject classifications (1970). Primary 35C10; Secondary 42A56, 33A 30, 33A 45 .

Key words and phrases. Poisson Szegö kernel, spherical harmonics, Jacobi polynomials, hypergeometric functions. 


$$
P_{m}^{(a, B)}(1)=\Gamma(\alpha+m+1)\{m ! \Gamma(\alpha+1)\}^{-1} .
$$

(E) For $p, q=0,1,2, \cdots ; n=2,3,4, \cdots$, and $z=r e^{i \theta} \in \mathrm{C}$, we set

$$
H_{n}^{p, q}(z)=D(p, q ; n) r^{|p-q|} e^{i(p-q) \theta} \frac{P_{\min (p, q)}^{(n-2,|p-q|)}\left(2 r^{2}-1\right)}{\omega_{2 n} P_{\min (p, q)}^{(n-2,|p-q|)}(1)} .
$$

(F) For $\lambda>-1 / 2$ and $m=0,1,2, \ldots, C_{m}^{\lambda}$ is the Gegenbauer polynomial of degree $m$ associated to $\lambda$ :

$$
C_{m}^{\lambda}(t)=\frac{\Gamma(2 \lambda+m) \Gamma(\lambda+1 / 2)}{\Gamma(2 \lambda) \Gamma(\lambda+m+1 / 2)} P_{m}^{(\lambda-1 / 2, \lambda-1 / 2)}(t)
$$

We have $C_{m}^{\lambda}(1)=\Gamma(2 \lambda+m)\{m ! \Gamma(2 \lambda)\}^{-1}$.

(G) For $k=0,1,2, \cdots ; n=3,4,5, \cdots$, and $t \in \mathbf{R}$ we set

$$
G_{n}^{k}(t)=\frac{d(k ; n)}{\omega_{n}} \frac{C_{k}^{(n-2) / 2}(t)}{C_{k}^{(n-2) / 2}(1)}
$$

(H) For $a, b \in \mathbf{R}, c>0$, and $|t|<1, F(a, b, c ; t)$ is the hypergeometric function of $t$ associated to $(a, b, c)$ :

$$
F(a, b, c ; t)=\sum_{n=0}^{\infty} \frac{\Gamma(a+n) \Gamma(b+n) \Gamma(c)}{\Gamma(a) \Gamma(b) \Gamma(c+n)} \frac{t^{n}}{n !} .
$$

If $c>a+b, \lim _{t \rightarrow 1^{-}} F(a, b, c ; t)$ exists and equals

$$
F(a, b, c ; 1)=\Gamma(c) \Gamma(c-a-b)\{\Gamma(c-a) \Gamma(c-b)\}^{-1} .
$$

(I) For $p, q=0,1,2, \cdots ; n=2,3,4, \cdots$, and $0 \leq r \leq 1$, we set

$$
S_{n}^{p, q}(r)=r^{p+q} \frac{F\left(p, q, p+q+n ; r^{2}\right)}{F(p, q, p+q+n ; 1)} .
$$

2. The Poisson kernel. We recall some facts from classical potential theory (cf. Stein-Weiss [8]). Let $B_{n}$ be the open unit ball in $\mathbf{R}^{n}, \Omega_{n}=$ $\partial B_{n}$ the unit sphere, and $\Delta$ the Laplace operator. Given $f \in C\left(\Omega_{n}\right)$, the Dirichlet problem of finding $u \in C\left(\bar{B}_{n}\right)$ such that $\Delta u=0$ in $B_{n}$ and $u \mid \Omega_{n}$ $=f$ is solved by the formula 


$$
u(x)=\int_{\mathbf{\Omega}_{n}} P_{n}(x, \xi) f(\xi) d \xi,
$$

where $P_{n}$ is the Poisson kernel defined for $(x, \xi) \in B_{n} \times \Omega_{n}$ by

$$
P_{n}(x, \xi)=\left(1-|x|^{2}\right) / \omega_{n}|x-\xi|^{n} .
$$

If $n \geq 3$, the orthogonal projection $\pi_{k}$ of $L^{2}\left(\Omega_{n}\right)$ onto the space of spherical harmonics of degree $k$ is given by

$$
\pi_{k} f(\eta)=\int_{\mathbf{\Omega}_{n}} G_{n}^{k}(\eta \cdot \xi) f(\xi) d \xi
$$

where $\eta \cdot \xi$ denotes the standard scalar product of $\eta$ and $\xi$ in $\mathbf{R}^{n}$. It is well known, and can be shown by the methods used below, that $P_{n}(n \geq 3)$ has the following expansion in spherical harmonics:

$$
P_{n}(\eta, \xi)=\sum_{k=0}^{\infty} r^{k} G_{n}^{k}(\eta \cdot \xi) \quad\left(0 \leq r<1 ; \eta, \xi \in \Omega_{n}\right) .
$$

If $n=2$, the corresponding formula is of course

$$
P_{2}\left(r e^{i \theta}, e^{i \phi}\right)=\sum_{k=-\infty}^{\infty} r^{|k|} e^{i k(\theta-\phi)}=1+2 \sum_{k=1}^{\infty} r^{k} \cos k(\theta-\phi),
$$

where we have identified $\mathbf{R}^{2}$ with $\mathbf{C}$.

3. The Poisson-Szegö kernel. We now consider $B_{2 n}$ and $\Omega_{2 n}$ as the unit ball and unit sphere in $C^{n}$, and we denote by $\Delta_{B}$ the Laplace-Beltrami operator associated to the Bergman metric on $B_{2 n}$. We have

$$
\Delta_{B}=\frac{4}{n+1}\left(1-|z|^{2}\right) \sum_{i, j=1}^{n}\left(\delta_{i j}-z_{i} \bar{z}_{j}\right) \frac{\partial^{2}}{\partial z_{i} \partial \bar{z}_{j}} .
$$

$\Delta_{B}$ is the basic invariant differential operator on the symmetric space $B_{2 n} \simeq S U(n, 1) / U(n)$. Although $\Delta_{B}$ degenerates at the boundary, the Dirichlet problem of finding $u \in C\left(\bar{B}_{2 n}\right)$ such that $\Delta_{B} u=0$ in $B_{2 n}$ and $u \mid \Omega_{2 n}=f$ is well posed for $f \in C\left(\Omega_{2 n}\right)$, and the solution is given by

$$
u(z)=\int_{\mathbf{\Omega}_{2 n}} \mathcal{P}_{n}(z, \zeta) f(\zeta) d \zeta,
$$

where $\mathcal{P}_{n}$ is the Poisson-Szegö kernel defined for $(z, \zeta) \in B_{2 n} \times \Omega_{2 n}$ by

$$
\mathcal{P}_{n}(z, \zeta)=\left(1-|z|^{2}\right)^{n} / \omega_{2 n}|1-z \cdot \zeta|^{2 n} .
$$


(Here, and in the sequel, $z \cdot \zeta$ denotes the Hermitian scalar product of $z$ and $\zeta$ in $\mathrm{C}^{n}$.) The uniqueness of the solution $u$ follows from the mean value property of $\Delta_{B}$.

For the proofs of the foregoing facts, see Stein [7], Hua [4], and Helgason [3].

The object of this note is to derive the analogue of the formula (1) for $\mathcal{P}_{n}(n \geq 2)$. (If $n=1, \Delta_{B}=1 / 2\left(1-|z|^{2}\right)^{2} \Delta$ and $\mathcal{P}_{1}=P_{2}$, so the formula in this case is simply (2).) Henceforth we assume $n \geq 2$.

Since $\Delta_{B}$ is invariant under $U(n)$ but not under $O(2 n)$, the appropriate analogue of spherical harmonics is the decomposition of $L^{2}\left(\Omega_{2 n}\right)$ into its $U(n)$-irreducible components. This theory is developed in Ikeda [5], VilenkinŠapiro [9], Folland [2], and Koornwinder [6], and the principal result is as follows.

Proposition 1. $L^{2}\left(\Omega_{2 n}\right)=\bigoplus_{p, q=0}^{\infty} \mathcal{S}^{p, q}$, where $\mathcal{F}^{p, q}$ is the space of restrictions to $\Omega_{2 n}$ of harmonic polynomials $f(z, \bar{z})$ on $\mathbf{C}^{n}$ which are homogeneous of degree $p$ in $z$ and degree $q$ in $\bar{z}$. $\mathcal{S}^{p, q}$ is $U(n)$-irreducible, and its dimension is $D(p, q ; n)$.

To identify the orthogonal projection onto $\sqrt{2} p, q$, we use the formula of Koornwinder [6]:

Proposition 2. If $f_{1}, f_{2}, \cdots, f_{D(p, q ; n)}$ is any orthonormal basis for $5^{p, q}$, then

$$
\sum_{j=1}^{D(p, q ; n)} f_{j}(\zeta) \overline{f_{j}(\eta)}=H_{n}^{p, q}(\zeta \cdot \eta)
$$

Consequently, the orthogonal projection $\pi_{p, q}: L^{2}\left(\Omega_{2 n}\right) \rightarrow \mathcal{F}^{p, q}$ is given by

$$
\pi_{p, q} f(\zeta)=\int_{\mathbf{\Omega}_{2 n}} H_{n}^{p, q}(\zeta \cdot \eta) f(\eta) d \eta
$$

We now identify the $\Delta_{B}$-harmonic extensions of elements of $\mathscr{T}_{2} p, q$.

Proposition 3. If $f \in \mathcal{F}^{p, q}$, the (unique) solution of the Dirichlet problem $\Delta_{B} u=0$ in $B_{2 n}, u \mid \Omega_{2 n}=f$ is

$$
u(r \zeta)=S_{n}^{p, q}(r) f(\zeta) \quad\left(0 \leq r \leq 1, \zeta \in \Omega_{2 n}\right)
$$

Proof. One element of $S_{2}^{p, q}$ is the restriction $f_{0}$ to $\Omega_{2 n}$ of $F_{0}(z)=$ $z_{1}^{p} z_{2}^{q}$. Since $\mathscr{S}_{2} p, q$ is irreducible, every element of $\mathscr{S}_{2}^{p, q}$ is a linear combination of translates of $f_{0}$ by elements of $U(n)$. Since moreover $\Delta_{B}$ 
commutes with the action of $U(n)$, it will suffice to prove the proposition for $f=f_{0}$. Thus we seek a $\Delta_{B}$-harmonic function on $B_{2 n}$ of the form $u(z)=$ $g\left(r^{2}\right) z_{1}^{p} \bar{z}_{2}^{q}$ where $r=|z|$. (We choose $g$ to depend on $r^{2}$ instead of $r$ because $u(z)$ must be smooth at $z=0$.) A straightforward computation shows that

$$
\begin{aligned}
\frac{\partial^{2} u}{\partial z_{i} \partial \bar{z}_{j}^{*}}= & {\left[g^{\prime \prime}\left(r^{2}\right) z_{i} \bar{z}_{j}+\delta_{i j} g^{\prime}\left(r^{2}\right)\right] z_{1}^{p} \bar{z}_{2}^{q} } \\
& +\delta_{i 1} p g^{\prime}\left(r^{2}\right) z_{j} z_{1}^{p-1} \bar{z}_{2}^{q}+\delta_{j 2} q g^{\prime}\left(r^{2}\right) \bar{z}_{i} z_{1}^{p} \bar{z}_{2}^{q-1}+\delta_{i 1} \delta_{j 2} p q g\left(r^{2}\right) z_{1}^{p-1} \bar{z}_{2}^{q-1} .
\end{aligned}
$$

Hence

$$
\sum_{i=1}^{n} \frac{\partial^{2} u}{\partial z_{i} \partial \bar{z}_{i}}=\left[r^{2} g^{\prime \prime}\left(r^{2}\right)+(n+p+q) g^{\prime}\left(r^{2}\right)\right] z_{1}^{p} \bar{z}_{2}^{q}
$$

and

$$
\sum_{i, j=1}^{n} z_{i} \bar{z}_{j} \frac{\partial^{2} u}{\partial z_{i} \partial \bar{z}_{j}}=\left[r^{4} g^{\prime \prime}\left(r^{2}\right)+(p+q+1) r^{2} g^{\prime}\left(r^{2}\right)+p q g\left(r^{2}\right)\right] z_{1}^{p} \bar{z}_{2}^{q}
$$

so that

$$
\begin{aligned}
\Delta_{B} u=\frac{4}{n+1}\left(1-r^{2}\right) z_{1}^{p} \frac{z_{2}^{q}}{2}\left[r^{2}\left(1-r^{2}\right) g^{\prime \prime}\left(r^{2}\right)\right. & \\
+ & \left.\left\{(p+q+n)-(p+q+1) r^{2}\right\} g^{\prime}\left(r^{2}\right)-p q g\left(r^{2}\right)\right] .
\end{aligned}
$$

Therefore if $\Delta_{B} u=0, g$ must satisfy

$$
t(1-t) g^{\prime \prime}(t)+[(p+q+n)-(p+q+1) t] g^{\prime}(t)-p q g(t)=0 .
$$

But this is the hypergeometric equation with parameters $a=p, b=q$, $c=p+q+n$, and the only solutions which are smooth at $t=0$ are multiples of $F(p, q, p+q+n ; t)$. Thus, writing $z=r \zeta$ with $\zeta \in \Omega_{2 n}$,

$$
u(z)=c F\left(p, q, p+q+n ; r^{2}\right) z_{1}^{p} \bar{z}_{2}^{q}=c F\left(p, q, p+q+n ; r^{2}\right) r^{p+q} f_{0}(\zeta),
$$

and the requirement that $u \mid \Omega_{2 n}=f$ shows that $c=\{F(p, q, p+q+n ; 1)\}^{-1}$. This completes the proof.

Remark. Except in the trivial cases $p=0$ or $q=0$ (when $\left.F\left(p, q, p+q+n ; r^{2}\right) \equiv 1\right), S_{n}^{p, q}(r)$ has a branch point at $r=1$. Thus, in contradistinction to the real case, the $\Delta_{B}$-harmonic extension of $f \in \mathcal{F}_{2} p, q$ to $B_{2 n}$ cannot be analytically continued outside $B_{2 n}$. This is not surpris- 
ing since the boundary is "at infinity" from the point of view of the Bergman metric.

We now state the main result.

Theorem. For $0 \leq r<1$ and $\eta, \zeta \in \Omega_{2 n}$,

$$
\mathcal{P}_{n}(r \eta, \zeta)=\sum_{p, q=0}^{\infty} S_{n}^{p, q}(r) H_{n}^{p, q}(\eta \cdot \zeta)
$$

The series on the right converges absolutely and uniformly for $\eta, \zeta \in \Omega_{2 n}$ and $0 \leq r \leq \rho$ for each $\rho<1$.

Proof. We first draw some consequences from Proposition 2. Let $f_{1}, \cdots, f_{D(p, q ; n)}$ be any orthonormal basis for $\mathscr{F}^{p, q}$; then, first,

$$
H_{n}^{p, q}(1)=H_{n}^{p, q}(\eta \cdot \eta)=\sum_{j=1}^{D(p, q ; n)}\left|f_{j}(\eta)\right|^{2}
$$

for any $\eta \in \Omega_{2 n}$. Second,

$$
\begin{aligned}
H_{n}^{p, q}(1) & =\omega_{2 n}^{-1} \int_{\Omega_{2 n}} H_{n}^{p, q}(1) d \eta \\
& =\omega_{2 n}^{-1} \sum_{j=1}^{D(p, q ; n)} \int_{\Omega_{2 n}}\left|f_{j}(\eta)\right|^{2} d \eta=\omega_{2 n}^{-1} D(p, q ; n) .
\end{aligned}
$$

Third, by orthogonality of the $f_{j}$ 's,

$$
\begin{aligned}
\int_{\Omega_{2 n}}\left|H_{n}^{p, q}(\eta \cdot \zeta)\right|^{2} d \eta & =\sum_{j=1}^{D(p, q ; n)} \int_{\Omega_{2 n}}\left|f_{j}(\eta)\right|^{2}\left|f_{j}(\zeta)\right|^{2} d \eta \\
& =\sum_{j=1}^{D(p, q ; n)}\left|f_{j}(\zeta)\right|^{2}=H_{n}^{p, q}(1)=\omega_{2 n}^{-1} D(p, q ; n) .
\end{aligned}
$$

Finally, $H_{n}^{p, q}(\eta \cdot \zeta)$ is Hermitian-symmetric in $\eta$ and $\zeta$ and is in $\zeta_{q}^{p, q}$ as a function of $\eta$ for each fixed $\zeta$; therefore by the Schwarz inequality,

$$
\left|H_{n}^{p, q}(\eta \cdot \zeta)\right|=\left|\int_{\Omega_{2 n}} H^{p, q}(\eta \cdot \xi) H_{n}^{p, q}(\xi \cdot \zeta) d \xi\right| \leq \omega_{2 n}^{-1} D(p, q ; n) .
$$

Moreover, since $\bigoplus_{p+q=k^{5}}$ is the space of all spherical harmonics of degree $k$, 


$$
D(p, q ; n) \leq d(p+q ; 2 n) \leq \text { const }(p+q+1)^{2 n} \text {. }
$$

Now since $F(p, q, p+q+n ; t)$ is an increasing function for $t \in[0,1]$, we have $\left|S_{n}^{p, q}(r)\right| \leq r^{p+q}$. Thus if $r \leq \rho<1$,

$$
\begin{aligned}
\sum_{p, q=0}^{\infty}\left|S_{n}^{p, q}(r) H_{n}^{p, q}(\eta \cdot \zeta)\right| & \leq \text { const } \sum_{p, q=0}^{\infty} \rho^{p+q}(p+q+1)^{2 n} \\
& =\text { const } \sum_{k=0}^{\infty} \rho^{k}(k+1)^{2 n+1}<\infty .
\end{aligned}
$$

This establishes the second assertion.

In particular, the right-hand side of (3) converges uniformly to a continuous function of $\zeta$ for each fixed $r \in[0,1)$ and $\eta \in \Omega_{2 n}$. Thus to prove (3) it will suffice to show that for each $r \in[0,1), \eta \in \Omega_{2 n}$, and $f \in C\left(\Omega_{2 n}\right)$,

$$
\int_{\Omega_{2 n}} \mathcal{P}_{n}(r, \zeta) f(\zeta) d \zeta=\int_{\Omega_{2 n}} \sum_{p, q=0}^{\infty} S_{n}^{p, q}(r) H_{n}^{p, q}(\eta \cdot \zeta) f(\zeta) d \zeta
$$

But on interchanging the summation and integration, we see from Propositions 2 and 3 that this is true when $f$ is a finite sum of spherical harmonics, and since such functions are dense in $C\left(\Omega_{2 n}\right)$, it is true in general. The proof is complete.

\section{REFERENCES}

1. A. Erdélyi et al., Higher transcendental functions. Vols. I, II, McGraw-Hill, New York, 1953. MR 15, 419.

2. G. B. Folland, The tangential Cauchy-Riemann complex on spheres, Trans. Amer. Math. Soc. 171 (1972), 83-133. MR $46 \# 8266$.

3. S. Helgason, Differential geometry and symmetric spaces, Pure and Appl. Math., vol. 12, Academic Press, New York, 1962. MR 26 \#2986.

4. L. K. Hua, Harmonic analysis of functions of several complex variables in the classical domains, Science Press, Peking, 1958; English transl., Transl. Math. Monographs, vol. 6, Amer. Math. Soc., Providence, R.I., 1963. MR 23 \#A3277; $30 \# 2162$.

5. M. Ikeda, On spherical functions for the unitary group. I. General theory, Mem. Fac. Engrg. Hiros hima Univ. 3 (1967), 17-29. MR 36 \#4042.

6. T. H. Koornwinder, The addition formula for Jacobi polynomials. II, Math. Centrum Amsterdam Afd. Toege p. Wisk. Report TW 133, 1972.

7. E. M. Stein, Boundary behavior of holomorphic functions of several complex variables, Princeton Univ. Press, Princeton, N.J., 1972. 
8. E. M. Stein and G. Weiss, Introduction to Fourier analysis on Euclidean spaces, Princeton Math. Ser., no. 32, Princeton Univ. Press, Princeton, N.J., 1971. MR $46 \# 4102$.

9, N. Ja. Vilenkin and R. L. Ša piro, Irreducible representations of the group $S U(n)$ of class I relative to $S U(n-1)$, Izv. Vysš. Učebn. Zaved. Matematika 1967, no. 7 (62), 9-20. (Russian) MR $36 \# 2741$.

DE PARTMENT OF MATHEMATICS, UNIVERSITY OF WASHINGTON, SEATTLE, WASHINGTON 98195 